\section{P057 ARTEMETHER SUPPRESSES MIGRATION AND INVASION OF FIBROBLAST-LIKE SYNOVIOCYTES THROUGH INHIBITION OF THE RHO SIGNALING PATHWAY IN RHEUMATOID ARTHRITIS}

J Jing, J-W Wang, Ma J-D, L-F Chen, L Dai*. Rheumatology, Sun Yat-sen Memorial Hospital, Sun Yat-sen University, Guangzhou, China

10.1136/annrheumdis-2018-EWRR2019.49

Career situation of first and presenting author Student for a master or a $\mathrm{PhD}$.

Introduction Experiments on collagen-induced arthritis rats showed that artemether, a new antimalarial drug derived from artemisinin, can reduce inflammatory cell infiltration, tissue edema, and bone erosion in the paws, which implied its potential efficacy in rheumatoid arthritis (RA). Fibroblast-like synoviocytes (FLS) in RA manifest tumor-like properties including increased adherence and invasiveness of adjacent cartilage and bone, resulting in joint destruction. However, effects of artemether on the aggressive properties of RA-FLS have not yet been elucidated.

Objectives The aim of this study was to investigate the role and underlying mechanism of artemether on the migration and invasion of RA-FLS.

Methods Synovial tissues were obtained by closed needle biopsy from 6 active RA patients and FLS were isolated and cultured in vitro. RA-FLS were treated with artemether at various concentrations, while methotrexate (MTX) and hydroxychloroquine (HCQ) were employed as controls. Cell viability, proliferation, cell-cycle, apoptosis, migration, invasion, and pseudopodium formation of RA-FLS were assessed by CCK-8 assays, EdU staining, Annexin V-FITC/PI staining, transwell assays, or F-actin staining respectively. The protein expression of RhoA, Rac1, and CDC42 were measured by Western blot.

Results

1. The $\mathrm{IC}_{50}$ value of artemether, MTX and HCQ on RA-FLS were $988 \mu \mathrm{M}, 181 \mathrm{nM}$ and $5433 \mu \mathrm{M}$, respectively.

Compared with untreated group, $\mathrm{IC}_{5}-20 \mu \mathrm{M}$ of artemether, $\mathrm{IC}_{5}-10 \mathrm{nM}$ of MTX and $\mathrm{IC}_{5}-20 \mu \mathrm{M}$ of HCQ showed no effect on the proliferation, cell-cycle or apoptosis of RA-FLS.

2. The transwell assays of migration and invasion showed that artemether $(20 \mu \mathrm{M})$ significantly inhibited migration and invasion of RA-FLS and reduced lamellipodia and filopodia formations. Under the same $\mathrm{IC}_{5}$ concentration, artemether $(20 \mu \mathrm{M})$ showed similar inhibitory effects as MTX $(10 \mathrm{nM})$, while HCQ $(20 \mu \mathrm{M})$ showed no effect on migration and invasion of RA-FLS.

3. Rho family proteins play important roles in modulating cell migration and invasion. Western blot analysis showed that artemether $(20 \mu \mathrm{M})$ and MTX $(10 \mathrm{nM})$ significantly reduced the expression of RhoA, Rac1, and CDC42 in RA-FLS, which indicated that artemether could inhibit the Rho signaling pathway.

Conclusions Artemether can suppress the migration and invasion capability of RA-FLS through inhibiting the Rho signaling pathway.

Acknowledgements This work was supported by National Natural Science Foundation of China (no. 81671612), Guangdong Natural Science Foundation (no. 2017A030313576, 2017A030310236) and Scientific Program of Traditional Chinese Medicine Bureau of Guangdong Province (no. 20161058, 20181058).

Disclosure of Interest None declared.

\section{P058 \\ CHEMOKINE CCL18 ENRICHED IN SYNOVIAL FLUID IS INVOLVED IN JOINT DESTRUCTION THROUGH PROMOTING MIGRATION OF FIBROBLAST-LIKE SYNOVIOCYTES IN RHEUMATOID ARTHRITIS}

${ }^{1} \mathrm{Y}-\mathrm{Q}$ Mo, ${ }^{2} \mathrm{G}-\mathrm{C}$ Zhang, ${ }^{1} \mathrm{~J}$ Jing, ${ }^{1} \mathrm{~J}-\mathrm{D} \mathrm{Ma},{ }^{1} \mathrm{~L}$ Dai ${ }^{*} .{ }^{1}$ Rheumatology, Sun Yat-sen Memorial Hospital, Sun Yat-sen University; ${ }^{2}$ Zhongshan School of Medicine, Guangzhou, China

10.1136/annrheumdis-2018-EWRR2019.50

Career situation of first and presenting author Young investigator.

Introduction CC chemokine ligand 18 (CCL18) which is either constitutively expressed or induced in monocytes/macrophages and dendritic cells has been reported to be highly expressed in peripheral blood and synovial fluid of rheumatoid arthritis (RA) patients compared with healthy controls, indicating the possible role of CCL18 in the development and pathogenesis of RA.

Objectives To explore the association of serum and synovial fluid CCL18 with clinical and radiographic outcome in RA and its potential effect on RA fibroblast-like synoviocytes (FLS).

Methods Consecutive patients with active RA (DAS28CRP>2.6) were recruited. Synovial fluid was collected from inflamed joints if available. Demographic and clinical data were collected according to the 2017 EULAR recommendation. Serum and synovial fluid CCL18 was detected by ELISA. RA-FLS was cultured in vitro with RA synovial fluid and neutralizing antibody to CCL18. Migration/invasion ability was analyzed by Transwell assay.

Results

1. Among 83 RA patients, age (median and IQR, similarly hereinafter) was $50(41 \sim 58)$ years old and 65 patients $(78 \%)$ were female, with median disease duration 36 (12 102) months, median DAS28-CRP 5.0 (4.6 6.1).

2. Serum CCL18 was $107 \quad(80 \sim 126) \mathrm{ng} / \mathrm{mL}$, which was significantly higher than healthy controls $[n=25,51(29 \sim 70)$ $\mathrm{ng} / \mathrm{mL}, \mathrm{p}<0.001]$. Serum CCL18 correlated slightly but significantly with CRP $(r=0.385, p<0.001)$, ESR $(r=0.239$, $\mathrm{p}=0.03), \operatorname{PrGA}(\mathrm{r}=0.249, \mathrm{p}=0.03)$, DAS28-CRP $(\mathrm{r}=0.368$, $\mathrm{p}=0.001)$, DAS28-ESR $(\mathrm{r}=0.336, \mathrm{p}=0.003)$, SDAI $(\mathrm{r}=0.360$, $\mathrm{p}=0.001)$, CDAI $(\mathrm{r}=0.328, \mathrm{p}=0.004)$ and HAQ $(\mathrm{r}=0.325$, $\mathrm{p}=0.004)$.

3. Synovial fluid CCL18 of 31 patients was 719 (415 1271) $\mathrm{ng} / \mathrm{mL}$, which was significantly higher than corresponding serum level (Paired test, $\mathrm{p}<0.001$ ). Among them, 13 patients who were treated according to treat to target strategy received X-ray assessment of hand/wrist both at baseline and month 12. Six patients who had one-year radiographic progression (a change of the Sharp/van der Heijde modified sharp score $\geq 0.5$ units) showed higher synovial fluid CCL18 than other 7 patients without radiographic progression [1481 (1244 2034) ng/mL vs. 458 (405 681) ng/mL, $\mathrm{p}=0.004)$.

4. When incubated with RA synovial fluid, the migration ability of RA-FLS was significantly increased; but this effect was Inhibited by neutralizing antibody to CCL18.

Conclusions CCL18 elevates especially in synovial fluid of RA patients, which may correlate with one-year radiographic progression through promoting migration of RAFLS.

Disclosure of Interest None declared. 